\title{
Network Selection for Autonomous Mobile Devices
}

\author{
Toktam Mahmoodi \\ Centre for Telecommunications Research \\ King's College London, U.K. \\ \{toktam.mahmoodi\}@kcl.ac.uk
}

\begin{abstract}
The rise of mixed mobile networks of WiFi, cellular small cells and traditional base stations, opens new challenges in optimising user's access to the networks. In fact, this mix of Radio Access Networks (RAN) become more heterogeneous as various technologies such as LTE, UMTS and WiMAX operates at the small cells. To fully utilise the capacity of such rich field of heterogeneous wireless connectivity, mobile devices should be able to select RAN (or a combination of RANs) for their connection and depending on their need. The proposed algorithm here is based on an autonomous agent at the mobile node, and assisted by integration of distributed cloud services. The ultimate aim is the design of RAN selection algorithm owned by autonomous and intelligent user agents that can significantly improve the user's Quality of Experience (QoE) in terms of network coverage, data rate, and battery life-time.
\end{abstract}

Index Terms-heterogenous mobile networks; network selection; mobile cloud computing; meta learning;

\section{INTRODUCTION}

With the growing demand for mobile data, mobile operators are increasingly turning to small cells (e.g. femtocells) to deal with the capacity crunch in dense urban areas and to add coverage in areas with low or zero cellular signal levels. To increase the capacity, not only licensed spectrum are exploited, but also service providers are looking to use e.g., WiFi to augment their investment in small cells. Emergence of small cells and their heterogeneity adds new dimensions to optimising users' access to these various Radio Access Networks (RANs). Soon users will be able to travel in a rich field of wireless networks with access to all wireless infrastructures around them, which will enable lower-cost connectivity and broader coverage. In fact, today's smartphones are armed with multiple radios, ad they will soon be capable of connecting to several networks at the same time, opening up enormous capacity and coverage.

These new opportunities comes with great challenges including selection of a RAN (or a combination of RANs) by the mobile user so as to receive the desired service at a lower cost and with an enhanced battery life-time. To this end, novel selection techniques should be deployed at the mobile devices. Intelligent and autonomous mobile devices should be able to explore their environment, find the RAN(s), and choose which one(s) to connect to so as to best meet their need. There have been efforts in the autonomous selection of RANs in heterogeneous environments using learning-based techniques [1], [2]. Market-based algorithm are also studied for the purpose of selecting the best available RAN [3], [4]. These algorithms mostly run at the mobile node side, while the proposed technique in [5], design two entities and at both the mobile side and in the core network. The entity at the mobile device prioritise available RANs based on the requirements of running application, and the final decision for selection RAN is made at the core network. Nonetheless, one of the remaining major challenges of network selection in the largely heterogeneous scenarios of today's mobile network is the consideration of numerous system parameters, which is in fact the common issue in the above mentioned research works. This problem, so far, has been addressed by reducing scenarios to the specific types of RANs or by limiting performance metrics to be optimised.

In this paper, we propose adaptive network selection strategies, based on an intelligent agent at the mobile device. The proposed algorithm here, introduces a unified framework for addressing all aspects of network selection and is based on two main novel ideas: enabling simultaneous, split connection over multiple wireless interfaces, and integration of distributed cloud services. These ideas are further discussed in section II. The design and architecture of the algorithm, different entities and their various technical challenges are elaborated in Section III, while summary of the proposed techniques, and the road ahead is explained in Section IV.

\section{Proposed Network Selection}

The proposed adaptive network selection here is based on an intelligent agent at the mobile device. This agent collects data based on estimation from the wireless environment, and also shared information between mobile users via a common entity in the cloud. The cloud-based entity is added mainly to address the challenges of dealing with large data sets of today's heterogeneous networks. The ultimate aim is the design of algorithms for autonomous and intelligent mobile devices that can select RAN(s) based on their own need and prediction/estimation from the wireless environment as well as the collaborative observations gathered by other mobile users. Hence, the RAN selection algorithm will be divided into two parts. While the first part is located in the cloud, the second part is located at each individual mobile node. The selection algorithms at the mobile will be designed so as to perform in the absence of access to the cloud while accessing to the shared experience in the cloud will improve the selection's efficiency. The main novelty of this technique lies in the following lines:

\section{A. Integration of cloud services}

To exploit the characteristics of available RANs and to avoid an excessive need for storage and processing power, new ways of coping with complexity are needed. Integration 
of the cloud computing concepts into the mobile environment, can overcome the obstacles related to the networking environment and the performance improvement of mobile computing systems including battery life-time, bandwidth, and capacity. The mobile cloud infrastructure we assume here, is similar to those discussed in [6], where the computing platform is the proximate infrastructure of, for example other mobile users.

\section{B. Meta Learner}

Another novel aspect of this work is the use of "meta learning" at the mobile agent. While access to the cloud will allow the agent to make a more informed decision, the autonomous agent at the mobile node should be able to operate in the absence of such access. In "meta learning" the goal is to understand how learning can become flexible according to the environment. By taking the right bias dynamically (in contrast with the base learning, where the bias is fixed a priori), the efficiency of learning can improve significantly [7]. Another interesting issue that can be addressed in meta learning, is to combine individual predictions and obtain higher accuracy at meta-level. For example, stacked generalisation [8] is a common scheme that combines a number of different learning machines and form meta-data. Afterwards, meta-data is feeded to another learning algorithm that can predict which combinations of algorithms give better results.

\section{Stitching multiple RANs together}

The better utilisation of network resources as addressed by this RAN, goes far beyond the existing techniques by allowing small partitions of access networks capacities to be stitched together and shape an appropriate size communication pipe for the mobile, while the access networks can be of various technologies. Preliminary study on how joining multiple RANs can enhance capacity is reported in [9]. Nevertheless, this area lacks fundamental research in providing a unified frame work for network selection that can consider diverse characteristics of the RANs independent of their types and the number of requirements by the mobile user.

\section{ARChiteCTURE}

While an autonomous agent is located at the mobile device and runs the network selection algorithm, part of the algorithm runs at the mobile cloud to access the collaborative observations (Figure 1). This entity, is accessed by the mobile autonomous agent, either to update or to retrieve information. The detail functionalities of the mobile agent and the cloudbased entity are as follows:

\section{A. Entity at the mobile cloud}

The cloud-based entity is accessible by all mobile users, and keeps track of the QoS in connection to different available RANs: it is the QoS-machine (i.e. there will be one QoSmachine per RAN). The autonomous agents at the mobile devices provide information for QoS-machine so as to learn from the connection experience of different mobile users that are in different physical locations running various applications. One of the critically important features of a self-organising

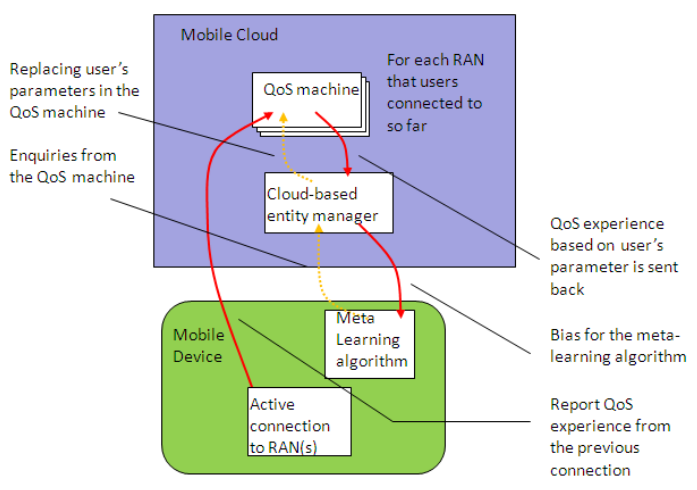

Fig. 1. Different entities and their communication flow in the network selection algorithm.

distributed system, similar to the small cell mobile network, is the explicit need for the estimation of environment parameters and possible changes in the environment. Therefore, similar techniques to the one discussed in [10] are needed to detect changes in the behaviour of the RANs and, if appropriate, training of the QoS-machine could be paused. For example, if a WiFi access point is switched off, its corresponding QoSmachine will not be modified until that access point comes back to life. Main challenges in the design of QoS-machine, and our approach to those challenges are discussed here:

A.1. Parametric learning techniques: One of the main challenges in the cloud-based entity is to consider the parameters such as physical location and application type that affect the QoS experience more significantly, while training the QoSmachine. Hence, novel parametric learning techniques will be needed. These parameters will be replaced by the agent at the mobile device each time the QoS-machine is either updated or being used, depending on the location and the individual need of mobile user.

A.2. The ensemble model: The newly instantiated QoSmachine at the mobile user can be the result of stitching multiple RANs together. Hence, a main challenge is how to combine features of different QoS-machines (correspond to different RANs) so as to best represent the ensemble properties. Another major question here is the prediction/estimation of which parameters can benefit the network selection algorithm, and which techniques should be used at the mobile terminal for predicting/estimating these parameters. Furthermore, as the selection algorithm runs at the mobile node, its computational complexity is also of great importance.

\section{B. Autonomous Agent at the Mobile Node}

At the mobile node, selection of RAN(s) depends on the QoS-machine as well as the mobile's estimation of the environment and the needs of its current application. Thus, the mobile node copies a selection of QoS-machines that best meet its need after replacing its own specific parameters predicted/estimated from its vicinity. In fact, the information from QoS-machines are used as a bias by the network selection algorithm at the mobile node. This approach is similar to 
the concept of "meta learning", where choosing the bias dynamically, and depending on the actual parameters of the environment, can improve the efficiency of the learning process [7]. The newly instantiated QoS-machine at the mobile user can be the result of stitching multiple RANs together. Hence, one of the main challenges is how to combine features of different QoS-machines (correspond to different RANs) so as to best represent the ensemble properties.

B.1. Estimation of the environment: A critically important feature of any self-organising distributed system, similar to the small cell mobile network, is the explicit need for the estimation of parameters of the environment and their possible changes. Therefore, designing a change detection mechanism similar to the one discussed in [10] is needed, so that changes in the behaviour of the RANs could be identified and, if appropriate, training of the QoS-machine could be paused. For example, if a WiFi access point is switched off, its corresponding QoS-machine won't be modified until that access point comes back to life. This will bring new questions including which specific behaviour of RANs can be examined for detecting such changes, and which techniques for detecting changes should be used.

B.2. Using meta learning at the mobile agent: At the mobile node, selection of RAN(s) depends on the QoS-machine as well as the mobile's estimation of the environment and the specific needs of its current application. Thus, the mobile node copies a selection of QoS-machines that best meet its need after replacing its own specific parameters predicted/estimated from its vicinity. In fact, the information from QoS-machines are used as a bias by the network selection algorithm at the mobile node. This approach is similar to the concept of "meta learning", where choosing the bias dynamically, and depending on the actual parameters of the environment, can improve the efficiency of the learning process [7]. On the other hand, if the mobile cloud can not be accessed, network selection algorithm performs but with reduced efficiency.

B.3. Shortcomings of the mobile devices: Despite the fact that smart phones are equipped with multiple wireless interfaces, simultaneous operation of these radios can be problematic. The small size of mobile device impose the radio circuits of e.g., WLAN and LTE to be located relatively close to each other, and thus they may affect each others functionality in simultaneous operation. One technique to address this challenge could be slicing the wireless resources and managing the simultaneous transmission on multiple interfaces within those slices [9]. Moreover, one of the major shortcomings in handling multiple connections is the design of the network stack at the mobile node. In the presence of multiple network interfaces, an application cannot take advantage of them to get higher throughput and at best it can only use the fastest connection. Furthermore, a connection oriented flow-like TCP cannot easily be handed over to a new interface, without reestablishing state and a user cannot easily and dynamically choose interfaces so as to minimise loss, delay, power consumption, or connection cost.

\section{SUMmary}

This paper, discuss the design of a radio access network selection algorithm based on an autonomous mobile agent. We mainly present our vision and the main concept of powering mobile devices to access the rich wireless field surrounding them autonomously. By integrating distributed cloud, and by allowing mobile to connect to multiple access networks at the same time, we introduce significant QoE enhancement.

To achieve such a design, two sets of challenges should be addressed. First, the design of appropriate learning techniques that can enhance the precision of estimated parameters, capture the user's QoE, and work efficiently with the meta data while operating relatively well without accessing those. The second set of challenges relate to the constraints of today's mobile devices in handling multiple wireless connections simultaneously, and the incapability of their software (or protocol stack) to handle data communication of a single application via multiple connections. The challenges and our approach to them are discussed in this paper.

Furthermore, the concept of network selection as discussed in this paper is motivated and in fact enabled, by the advancements in three major areas of mobile communications including, software defined radio, small cell network and mobile cloud computing. We assume mobile devices have multiple radio interfaces and cognitive and software defined radio techniques allow the mobile to explore their surrounding wireless spectrum. The small cell mobile network provides a rich platform of various RANs with different technologies, and hence different latencies, data rate and range of coverage. Finally, the existence of mobile cloud computing infrastructure allows our proposed algorithm to improve its efficiency with little extra computing and battery cost.

\section{REFERENCES}

[1] Y. Wang and K. Zhang, "Decision Tree based Unsupervised Learning to Network Selection in Heterogeneous Wireless Networks," in Consumer Communications and Networking Conf. (CCNC), Jan. 2011.

[2] H. Tabrizi, G. Farhadi, and J. Cioffi, "A Learning-Based Network Selection Method in Heterogeneous Wireless Systems," in Global Communications Conf. (GLOBECOM), Dec. 2011.

[3] D. Niyato and E. Hossain, "Dynamics of Network Selection in Heterogeneous Wireless Networks: An Evolutionary Game Approach," Trans. on Vehicular Technology, vol. 58, pp. 2008-2017, May 2009.

[4] S. Paris, F. Martignon., I. Filippini, and A. Capone, "A Truthful Auction for Access Point Selection in Heterogeneous Mobile Networks,' in Int'l Conf. on Communications (ICC), Jun. 2012.

[5] A. Kaloxylos, I. Modeas, F. Georgiadis, and N. Passas, "Network Selection Algorithm for Heterogeneous Wireless Networks: from Design to Implementation," Network Protocols and Algorithms, vol. 1, 2009.

[6] M. Satyanarayanan, P. Bahl, R. Caceres, and N. Davies, "The Case for VM-Based Cloudlets in Mobile Computing," Pervasive Computing, vol. 8, pp. 14-23, Oct. 2009.

[7] R. Vilalta and Y. Drissi, "A Perspective View and Survey of MetaLearning," Artificial Intelligence Review, vol. 18, pp. 77-95, Oct. 2002.

[8] D. H. Wolpert, "Stacked Generalization," Neural Networks, vol. 5, pp. 241-259, Mar. 1992.

[9] K.-K. Yap, T.-Y. Huang, M. Kobayashi, Y. Yiakoumis, N. McKeown, S. Katti, and G. Parulkar, "Making Use of All the Networks Around Us: A Case Study in Android," in SIGCOMM workshops, Aug. 2012.

[10] T. Mahmoodi and M. Roveri, "Identifying Network Failure via Detecting Changes in Power Profile," in Int'l Conf. on Pervasive Computing and Communications (PERCOM) Workshops, Mar. 2012. 